\title{
of chickens in the United States of America
}

\author{
avid A. Kennedy: dakso \\ Pennsylvania State University, University Park, PA, USA \\ East Lansing, \\ Pennsylvania State University, University Park, PA, 16802, USA
}

Marek's disease, a disease primarily affecting immature chickens, is a worldwide problem that has tudy the epidemiology of this disease is available because the United States Department of Agriculture has required mandatory inspections of all commercially sold poultry of significant ale since the mid-20th century with ov the number with Marek's disease in this category of birds. The objective of this study was to analyze temporal and spatial patterns in this condemnation data to gain insight into the ecology and epidemiology of the we tested for statistical significance using extended linear me analysis confirmed previous findings that there are differences in leukosis condemnation rates between stes, across years, and within years. The analysis also revealed several patterns not previously highlighted, including spatial and temporal autocorrelations in leukosis condemnation, changes to ver time. These patterns suggest that locally shared farm practices, virus transmission between also discuss the plausibility of other potential explanations for these patterns. 


\section{Introduction}

Chickens, Gallus gallus domesticus, currently account for about 35\% (Meyer and Steiner 2011) of the meat consumed in the United States of America (US) with this figure expected to exceed $40 \%$ by 2020 (United States Department of Agriculture 2011). The profitability of chicken farming, however, is under constant threat from disease outbreaks. Marek's disease (MD), one such disease that caused an economic crisis in the poultry industry in the 1960s (Biggs and Nair 2012; Davison and Nair 2005), is estimated to cost the global poultry farming industry over 1 billion USD annually (Morrow and Fehler 2004; Nair 2005). For such an important disease, however, surprisingly little is known about its epidemiology. Here, we study patterns in 52 years of publicly available data on MD-associated chicken condemnations to gain insights about the ecology of this highly infectious disease and its causative agent, Marek's disease virus (MDV). This dataset includes a total of 287 billion chickens, comprising over $99 \%$ of all young chickens processed in the US during this time (United States Department of Agriculture 2014).

Clinical signs of the disease include paralysis, immune suppression, tumor formation, and death. The impact of MD increased substantially during the industrialization of poultry farming that took place during the mid twentieth century. The profitability of the US chicken industry was in jeopardy until the first vaccine was introduced that protected chickens from developing MD (Okazaki et al. 1970). Since then, MD has been controlled primarily through vaccination. This method of control, however, is tenuous (Davison and Nair 2004), because the efficacy of two generations of MD vaccines have been reduced in the face of pathogen evolution (Witter 1997). It is therefore important to understand the dynamics of MD over space and time in order to predict and design optimal responses in the event that the efficacy of current vaccines also declines. Pathogen evolution leading to vaccine failure is a major concern for diseases of both humans and farmed animals (Mooi et al. 2001; Read and Mackinnon 2008; Welch et al. 2011). Relative to human systems, studying this evolution in agricultural systems has the benefit that the ecology can be carefully controlled and monitored. MD may therefore offer an important opportunity to study how the ecology of a disease contributes to vaccine failures.

In 1957, the United States Department of Agriculture began collecting data to monitor the health of the poultry farming industry. Since 1961, these data included the numbers of "young chickens" condemned for a condition called "leukosis." The leukosis complex is a category used by the Food Safety and Inspection Service (FSIS) that includes neoplastic diseases caused by MD, lymphoid leukosis, reticuloendotheliosis or lymphoproliferative disease in turkeys. Assignment to this condemnation category occurs when birds have at least one lesion that can be "grossly defended as a lesion of leukosis" (Food Safety and Inspection Service 2012). Based on age and species, the designation of leukosis in young chickens refers almost exclusively to MD (Sharma 1985; Food Safety and Inspection Service 2012). Inspection occurs at processing plants in the US, and so over $99 \%$ of all young chickens are inspected for leukosis (United States Department of Agriculture 2014). We analyzed this data on leukosis condemnation rates in young chickens to determine how MD prevalence varies over time and space. By variation over time, we are referring to patterns at three different timescales: directional changes that take place over many years, 
periodic cycles that take place yearly, and abrupt non-periodic changes that occur over the course of only a few months. By variation over space, we are referring to differences based on broad scale geographic patterns (e.g. northern vs. southern states), as well as correlations in disease incidence between nearby states versus more distant states.

Previous work using leukosis condemnation data were published in 1985 (Purchase 1985), in 1996 (Witter 1996), and in 2013 (Dunn and Gimeno 2013). These data have gained general acceptance as a measure of MD incidence. We expand on previous work by using seasonal trend decomposition (STL) to view changes in leukosis condemnation rates over time, and by using an extended linear model to quantitatively test for the importance of variation between states, over time, and throughout individual years on leukosis condemnation rate.

\section{Methods}

\subsection{Data collection and preprocessing}

The data used in this study were collected from the Poultry Slaughter reports available through the National Agricultural Statistics Service website (http:// usda.mannlib.cornell.edu). Data for these reports were collected by FSIS for all federally inspected poultry processing plants, approximately 300 poultry slaughter plants currently (United States Department of Agriculture 2014). Using these reports, the number of leukosis cases and total number of young chickens inspected in each month for each state were manually entered into an Excel file. The data were read into the $\mathrm{R}$ statistical programming language (R Development Core Team 2012) for further analyses.

As with many publicly available agricultural datasets, some data were unavailable to avoid disclosing data for individual operations. At the state-wide level, many data points were missing, and were treated as missing data. At the nation-wide level, however, many fewer data points were missing, and so values for these missing data were inferred using the 'na.approx' function in the 'zoo' package in R. This function used linear interpolation in time on log transformed leukosis condemnation rates to estimate values for months with missing values. In total four data points out of 639 total data points were inferred. We then used these data with STL analysis and extended linear modeling to explore historical patterns in MD-induced leukosis condemnation rates over space and over time.

\subsection{Statistical models}

STL analysis uses a loess smoother to decompose a time series into a seasonal component, a trend, and a remainder (Cleveland et al. 1990). These components of a time series can then be readily visualized using this technique. The benefit of STL analysis is that it extracts patterns in the data that might otherwise be difficult to see, but whether the patterns are statistically significant is not readily clear. This method can therefore serve to visualize time series, and it can be useful as a supplement to a difficult-to-visualize model that employs hypothesis testing.

Extended linear modeling is a generalization of a linear model that uses splines, or piecewise polynomials, to model nonlinear relationships between model predictors and responses 
(Huang and Stone 2003). Extended linear modeling is more appropriate for this study than traditional linear modeling, because the effect of time on leukosis condemnation is expected to be nonlinear due to changes in farm practices, chicken life history, and pathogen virulence. Extended linear modeling, just like traditional linear modeling, can readily be used for hypothesis testing (Huang and Stone 2003). The drawback of extended linear modeling, however, is that effect sizes are often difficult to interpret, because they act on spline-transformed predictors, rather than on the measured predictors themselves. This technique can therefore be complemented by the use of other techniques such as STL that make visualization of effect sizes trivial.

We first ran a STL on the log-transformed nationally aggregated data using the 'stl' function in $\mathrm{R}$ ( $\mathrm{R}$ Development Core Team 2012). The data were log transformed because fluctuations and changes in condemnation rates appeared to happen on a multiplicative scale.

Preliminary examination also revealed that the seasonal component changed over time. To allow for this variation, we set the parameter s.window to 25 . This value was chosen after examining diagnostic plots, as suggested by (Cleveland et al. 1990). Our main conclusions, however, are robust to other reasonable choices of this parameter. After examining the STL plots, we second fit an extended linear model to the state-wide aggregated data. The factors in this model included long-term temporal patterns, an annual temporal cycle, state-by-state variation, and all of the two-way interactions between these factors. Because the STL analysis showed that the effect of time on leukosis condemnation rate was nonlinear, we added flexibility to the linear model by converting time into a series of piecewise polynomials, or basis splines. Importantly, any smooth curve can be described as a linear combination of basis splines (Härdle 1990). In practice, this transformation was performed using the 'bs' function in the 'splines' package in R, with cubic splines and 5 degrees of freedom. We used 5 degrees of freedom, because fewer provided a visually poor fit to the data, and more did not substantially improve the $R^{2}$ value of the models.

The results of our STL analysis showed that MD dynamics fluctuated within years, with relatively high rates in the winter/fall and relatively low rates in the spring/summer, consistent with previous studies (Witter 1996; Walkden-Brown et al. 2013). Preliminary analysis also revealed that these fluctuations closely matched a sine curve. We were thus able to fit the amplitude $A$ and phase shift $\varphi$ in the data using the trigonometric identity $A$ $\sin (t+\varphi)=c_{1} \cos (t)+c_{2} \sin (t)$. The coefficients $c_{1}$ and $c_{2}$ were estimated with linear modeling techniques, and they were then back transformed to calculate the amplitude and phase shift of the annual cycles. We note that time $t$ in the above equation was rescaled to range from $\pi / 6$ in January to $2 \pi$ in December, ensuring that each cycle would have a period of one year.

We standardized the data by transforming them according to the following expression, $\log _{10}\left(\left(B_{L}+1\right) /\left(B_{T}+2\right)\right)$. Here, $B_{L}$ is the number of birds condemned for leukosis and $B_{T}$ is the total number of birds inspected. This transformation extends the range of proportion data, in our case, making it more suitable for use in linear models. We chose to transform our data, rather than to perform a logistic regression, because preliminary analysis revealed substantial data overdispersion, suggesting that a logistic regression could be inappropriate. We fit our models using the 'Im' function in R. The significance of each factor was 
determined through a likelihood ratio test comparing the full model to a model lacking the respective factor of interest.

\subsection{Statistical analysis}

We assessed whether leukosis condemnation rates were becoming more variable over time in three ways. First, we visually examined the seasonal component of leukosis condemnation from the STL analysis. Second, we used a likelihood ratio test to determine whether the absolute value of the remainder component from the STL analysis was increasing over time. Third, we calculated yearly coefficients of variation in the national leukosis condemnation rate data (leukosis condemnations divided by the total number of birds inspected). The advantage of using coefficients of variation as opposed to the variance was that coefficients of variation could be used to compare data with different means. Because leukosis condemnation rates changed substantially over time, this correction for different means was necessary. We used a linear model to determine the effect of time on the coefficient of variation, and we tested for the significance of time using a likelihood ratio test. To validate our extended linear model, we plotted the model predictions vs. the model residuals, which did not reveal any clear systematic failures in our model.

To assess state-specific patterns in leukosis condemnation rates and how these changed over time, we also analyzed the condemnation rate at each state at any given time point relative to the condemnation rate seen at the national scale at that same time point. For ease of interpretability, we then log-transformed these values, so that positive values indicate higher condemnation than the national average, and negative values indicate lower condemnation than the national average. Hereafter, these transformed data are referred to as the "relative leukosis condemnation rates." Within individual states, we tested for autocorrelations in the relative leukosis condemnation rates using the 'acf' function in R. We also tested for correlations between states in their relative leukosis condemnation rates, using a linear model with pairwise log-distances as the only factor. The accuracy and precision of correlation coefficient estimates between pairs of states depend on the number of times at which data were available for both focal states. Pairs of states with fewer than 100 overlapping data points were therefore excluded from this analysis. The significance of the effect of distance on the relative leukosis condemnation rate was determined using a likelihood ratio test.

\section{Results}

Consistent with previous studies, the STL analysis showed that the underlying trend in leukosis condemnation rate has changed over time with rates generally declining after an initial period of increase (fig. 1). Analysis of the extended linear model further showed that this change over time was statistically significant $\left(\chi^{2}=23502\right.$, d.f. $\left.=145, p<0.001\right)$. Presumably, the introductions of new MD vaccines have had substantial impacts on MD incidence, and so we interpret changes in leukosis condemnation rates in each vaccine era separately. First, from 1961 to 1970, the period before any MD vaccines were available (Davison and Nair 2004), we see that leukosis condemnation rates rose rapidly, increasing by more than tenfold before leveling off. Second, from 1971 to 1983, the period after the 
first generation Herpesvirus of Turkeys (HVT) vaccine was licensed and before the second generation bivalent vaccine was licensed (Witter 1985), leukosis condemnation rates declined to a slightly lower rate than that of the early 1960s. The majority of this decline took place over the first several years after the HVT vaccine was introduced. After this rapid decline, leukosis condemnation rates continued to decline, but at a much slower pace. Third, from 1984 to 1995, the period between when the bivalent vaccine was licensed and before the third generation attenuated virus Rispens vaccine was introduced in the USA (Morrow and Fehler 2004), leukosis condemnation rates continued to decline very slowly. Fourth, from 1996 onwards, after the Rispens vaccine was licensed, leukosis condemnation rates declined at a much faster rate. Care should be taken when attributing any decline to a particular vaccine, but this most recent decline is particularly curious because in the US, the Rispens vaccine is only rarely used on the category of "young chickens" that the data cover.

We found that in addition to the trend described above, leukosis condemnation rate fluctuated within years on average by 4.8 fold (fig. 1A). In contrast, between years, leukosis condemnation rate fluctuated on average by 1.4 fold, with the largest change between any two years being a 3.3 fold decrease between 2006 and 2007. This pattern demonstrates that leukosis condemnation rates can change extremely quickly but that over the long term, even with the emergence of more virulent virus strains and the introduction of improved vaccination methods, the national leukosis condemnation rate has changed only gradually (fig. 1C). Additionally, the coefficient of variation in leukosis condemnation rates within years increased over time $\left(\chi^{2}=10.9\right.$, d.f. $\left.=1, p<0.001\right)$. The seasonal component of the STL analysis reveals that some, but not all, of this increase was due to changes in the effect of seasonality over time. The amplitude of seasonal fluctuations indeed increased until about 1990, but then it began to decrease (fig. 1B). Meanwhile, the absolute value of the remainder component of the STL analysis has been steadily increasing (fig. 1D, $\chi^{2}=72.5, d . f .=1, p<$ 0.001 ). In other words, although leukosis condemnation rates have been generally declining, the yearly variation in leukosis condemnation rates has been increasing, and a large component of this increase is unexplained by changes in seasonal fluctuations.

States also differed in their baseline leukosis condemnation rates $\left(\chi^{2}=734.9\right.$, d.f. $=27, p<$ 0.001). In other words, some states had much higher leukosis condemnation rates than others. Notably, the relative leukosis condemnation rate between states was correlated with the distance between them (fig. $2, \chi^{2}=8.9, d_{\text {.f. }}=1, p=0.003$ ), such that states close together tended to have more similar condemnation rates that those further apart. States in the mid-Atlantic tended to have high relative leukosis condemnation rates while states in the south tended to have low rates (fig. S1). However, the change in leukosis rates over time differed between states $\left(\chi^{2}=4094.3\right.$, d.f. $\left.=130, p<0.001\right)$. Interestingly, within states there were strong autocorrelations in these rates (fig. S2), suggesting that changes in relative leukosis condemnation rates happened gradually rather than abruptly. This, in turn, implies that states with high relative leukosis condemnation rates will likely continue to have high rates in the near future, and likewise for states with low rates.

A visual comparison of the predictions of our extended linear model to the data showed that the model captured much of the dynamical change in leukosis condemnation rates across states over time (fig. 3). This visual similarity relates to an $R^{2}$ value of 0.93 . Importantly, 
our intent was not to exactly replicate the data, but rather to smooth out the stochastic fluctuations so that the general trends in the data might be more readily apparent. As can be seen in fig. 3, the same general temporal pattern in leukosis condemnation rates in the national data were found in the individual state data. We note, however, that although the general trend was the same, there were moderate differences in the timing, shape, and duration of these phases between states, as has previously been pointed out (Witter 1996). For example, leukosis condemnation rates peaked in Maryland and Delaware the late 1960s, whereas these rates peaked in Alabama and Tennessee in the early 1970s.

An additional feature of the data was that the amplitude of the annual cycles in condemnation rates differed substantially between states $\left(\chi^{2}=440.2\right.$, d.f. $\left.=60, p<0.001\right)$. For example, California, Washington, and Pennsylvania had small annual cycles relative to Georgia, Maryland, and Alabama. The amplitude of these cycles additionally changed over time $\left(\chi^{2}=499.2, d . f .=10, p<0.001\right)$. As fig. 3 shows, the relative amplitudes of the cycles were small initially, they increased after the first generation vaccine was introduced and they remained large until around the time the third generation Rispens vaccine was introduced, when they began to shrink. This change can also be readily seen at the national scale in fig. $1 \mathrm{~B}$.

\section{Discussion and Conclusions}

MD has been a threat to the profitability and sustainability of the chicken farming industry in the past, and if the current generation of MD vaccines were to lose efficacy, MD could once again emerge as a threat (Davison and Nair 2004). Here we used publicly available data on MD-induced leukosis condemnation rates in young chickens to confirm that leukosis condemnation rates have changed significantly over time, and that these dynamical changes differed between states. The analysis also shows that the amplitude of annual cycles differed between states, and that these amplitudes additionally changed over time.

As we pointed out earlier, in addition to gradual changes in the leukosis condemnation rate, rapid changes occurred within years (fig. 1). These rapid fluctuations suggest that seasonal weather and seasonal management practices, such as changes to ventilation and the timing of house cleanouts, may play key roles in the ecological dynamics of MD. Proper ventilation, regular house cleanouts, and other aspects related to hygiene such as filtering air are known to influence MD risk (Grunder et al. 1975). Perhaps the best example of this is specific pathogen free (SPF) flocks, where extremely rigorous hygiene and biosecurity practices are employed to keep flocks clean of various pathogens, including MDV (Luginbuhl 2000). This level of hygiene is of course economically infeasible at the production end of the poultry industry. Nevertheless, our analysis suggests that historical condemnation in winter months could have been reduced by on average $62 \%$ (between $35 \%$ and $78 \%$ depending on the year) if summer conditions and management practices were maintained year round. What is needed then is to determine the conditions and management practices which drive seasonal differences. Notably, with current levels of leukosis condemnation being so low, any changes to general management practice would likely be economically infeasible. Targeted changes on farms with recent history of high leukosis condemnations, however, might be practical. 
Changes in leukosis condemnation rate over a longer timescale (i.e. between years) were slow and gradual. One might expect to see rapid increases in condemnation rates when more virulent strains were emerging in the late 1970s and mid 1990s, and to see rapid decreases in condemnation rates when new vaccines were introduced in 1971, 1983, and 1996. For example, the bivalent vaccine that was introduced in 1983 reduced leukosis condemnation rates in broiler chickens by $78 \%$ relative to HVT vaccination (Witter et al. 1984), but a 78\% reduction in leukosis condemnation rate from the level observed in 1982 was not achieved until 1991. This can be partially explained by new vaccines being adopted by the industry only gradually. Additionally, this pattern may indicate that environmental reservoirs of virus might have buffered rapid changes to MD dynamics. Whether environmental reservoirs exist for this virus is, however, still an open question. If reservoirs exist, the observations that the risk of developing MD is dose dependent (Powell et al. 1980), the virus can persist for long periods outside of a host (Jurajda and Klimes 1970; Carrozza et al. 1973), and vaccination reduces the amount of virus shed into the environment by infected birds (Purchase and Okazaki 1971; Islam and Walkden-Brown 2007), suggest that the benefits of vaccination may accumulate over time as environmental reservoirs of virus diminish. The existence of such a reservoir is thus supported by the strong autocorrelation over time in relative leukosis condemnation rates within states.

The Rispens vaccine is typically only used for egg layer and broiler breeder chickens, which are longer lived than broiler chickens and thus at greater risk of developing clinical MD. Nevertheless, condemnation of young chickens due to leukosis has substantially decreased since the introduction of the Rispens vaccine. We believe that at least part of this decrease is likely not attributable to the Rispens vaccine, but rather, to the implementation of automated in ovo vaccination that was introduced at a similar time. Automated in ovo vaccination was introduced in 1995 as a more effective way to administer the second-generation bivalent vaccine (Sarma et al. 1995). This process improved both the reliability of administration of vaccines and the efficacy of vaccines (Ricks et al. 1999), and might have thus led to a decrease in leukosis condemnation. Nevertheless, the Rispens vaccine likely also contributed to this decrease in condemnation. Although not regularly used in broiler chickens, the Rispens vaccine was often used in flocks with a recent history of MD problems. Moreover, using a different vaccine in breeder hens and offspring makes interference between maternal antibodies and vaccines less likely (King et al. 1981), although at least one study failed to observe such a benefit (Dudnikov and Witter 2001).

More generally, vaccination undoubtedly played an important role in changing the leukosis condemnation rates over time, but the data also show changes to condemnation rates that cannot be easily explained by changes in vaccination policy, for example, decreases to condemnation rates that occurred even before the introduction of the HVT vaccine in 1971 (fig. 3). This suggests that changes to farming practices other than vaccination may have also played important roles in the decline of leukosis condemnation rates. During the 52year period that the data span, many changes to chicken rearing took place, such as improvements to ventilation systems (Sainsbury 2000). Perhaps the largest change was the increased growth rate of chickens that resulted from selective breeding and improvements to diet. Havenstein et al. (2003), for example, showed that compared to chickens from 1957 fed 
1957-type diets, chickens from 2001 fed 2001-type diets, were approximately four times larger at various time points post hatch. In practice, the increased growth rate translated to decreased time to slaughter, shortened from an average of several months in 1960 to an average of about six weeks today. Clinical signs of MD can sometimes take weeks to manifest (Schat and Nair 2008), and so this decrease in development time likely played an important role in the observed decrease in leukosis condemnation rates. Nevertheless, without having more details on how average time to slaughter changed over this time period, we cannot estimate how important this factor was in the observed changes to leukosis condemnation rate. We note, however, that the observed decrease in leukosis condemnation rate cannot be entirely explained by changes in growth rate, because we still see outbreaks of MD today in unvaccinated and undervaccinated flocks. Instead, the observed reduction in leukosis condemnation rate was likely due to a combination of factors, including decreased development time, improved vaccination, and increased genetic resistance to MD.

Problems with MD can occur quickly and without warning, particularly in flocks that have not been properly vaccinated. Additionally, the virus (Rispens et al. 1969, 1972) and antibodies against the virus (Chubb and Churchill 1968) can be detected in flocks showing no clinical signs. These lines of evidence have led to general speculation that MDV is ubiquitous among commercial farms in the US. Despite its apparent ubiquity, however, our analysis provides two lines of evidence consistent with the competing hypothesis that MDV is geographically clustered and locally transmitted. First, we found a strong correlation by distance of relative leukosis condemnation rates which would be expected if the virus moved readily between nearby farms (fig. 2). Second, leukosis condemnation rates within states were highly autocorrelated temporally which would be expected if there were substantial transmission of the MDV between flocks of chickens. These data, however, are not conclusive. Rather than being driven by local transmission, it is also biologically plausible that these patterns could be driven by factors that correlate with geography, such as farm density, litter availability, or weather. Changing litter between cohorts is commonly done to delay exposure, but in some geographic regions, litter is more easily replaced than in others, because of differences in both the supply of fresh bedding material and the demand for used litter for fertilizer. External temperatures may also indirectly affect MD risk, because in the interest of conserving heat, ventilation is often reduced during cold periods. Additionally, this pattern might be explained by locally shared farming practices rather than local virus transmission.

Our finding that the coefficient of variation in leukosis condemnation rates and the absolute value of the remainder component of the STL analysis increased over time suggests that the importance of sporadic changes in condemnation rate has increased. In other words, MD incidence has become more irregular in recent years. Because leukosis condemnation rates are quite low in these years, this increase in unpredictability may be driven by just a few small outbreaks. A study in Australia found that on many farms MDV is often not detectable (Walkden-Brown et al. 2013), and based on this observation, the authors conclude that because MD incidence is highest in the winter, the industry might be able to increase profits by vaccinating only during these high-risk periods. Our results, however, suggest that in the US, leukosis condemnation rates are becoming less predictable, and so this approach might backfire if used on US farms. 
As can be seen in figs. 1C and 3, the amplitude of seasonal variation in leukosis condemnation rate changed over time. It started out relatively low, increased during the HVT vaccine era, peaked during the bivalent vaccine era, and decreased in the Rispens vaccine era. This observation adds to a body of literature suggesting that vaccines can have complex effects on cyclic disease dynamics (Anderson et al. 1984; Rohani et al. 1999; Earn et al. 2000; Pitzer et al. 2009). Interestingly, Pitzer et al. (2011) found that intermediate coverage with a highly efficacious vaccine against rotavirus could lead to large amplitude cycles in disease incidence, but the amplitude of these cycles were dampened at both higher and lower levels of vaccine coverage. In the MDV system, large amplitude cycles were associated with intermediately efficacious vaccines being available, but smaller amplitude cycles occurred before vaccines were introduced and after the highly efficacious Rispens vaccine was introduced. This may be an analogous effect worth exploring further in a theoretical context.

An important point is that leukosis condemnation data provide limited information about MD and MDV. The specificity of leukosis condemnation data as a proxy for MD is likely high, because MD is the only substantial cause of leukosis in young chickens, but the sensitivity of this data as proxy for MD is likely imperfect for a variety of reasons, including that MD does not always induce leukosis, and that inspectors spend very little time with each bird inspected. Our main conclusions are likely robust to this imperfect sensitivity, because the results are derived from changes in condemnation rates, rather than the absolute condemnation rates. Even so, improvements to vaccination, shortened rearing periods, increased genetic resistance, and many other factors have presumably decoupled the link between the prevalence of MDV infection and leukosis condemnation rates. What is needed, therefore, is to combine surveillance and quantification of the virus on farms (WalkdenBrown et al. 2013) with mechanistic mathematical models (Atkins et al. 2013a,b) to learn about the mechanisms that govern viral dynamics. Nevertheless, analyses of leukosis condemnation data can be useful for generating hypotheses about historical and current drivers of MD dynamics.

\section{Supplementary Material}

Refer to Web version on PubMed Central for supplementary material.

\section{Acknowledgements}

We thank R. Witter, N. Mideo, and two anonymous reviewers for comments on earlier drafts. This work was funded by the RAPIDD program of the Science and Technology Directorate, Department of Homeland Security and Fogarty International Center, National Institutes of Health (DK, AR) and the Institute of General Medical Sciences (R01GM105244), National Institutes of Health as part of the joint NSF-NIH-USDA Ecology and Evolution of Infectious Diseases program (AR, PD). The funders had no role in study design, data collection and analysis, decision to publish, or preparation of the manuscript.

\section{References}

Anderson RM, Grenfell BT, May RM. Oscillatory fluctuations in the incidence of infectious disease and the impact of vaccination: time series analysis. Journal of Hygiene. 1984; 93:587-608. [PubMed: 6512259] 
Atkins KE, Read AF, Savill NJ, Renz KG, Islam AFM, Walkden-Brown SW, Woolhouse MEJ. Vaccination and reduced cohort duration can drive virulence evolution: Marek's disease virus and industrialized agriculture. Evolution. 2013a; 67:851-860. [PubMed: 23461333]

Atkins KE, Read AF, Walkden-Brown SW, Savill NJ, Wool-house MEJ. The effectiveness of mass vaccination on Marek's disease virus (MDV) outbreaks and detection within a broiler barn: A modeling study. Epidemics. 2013b; 5:208-217. [PubMed: 24267877]

Biggs PM, Nair V. The long view: 40 years of Marek's disease research and avian pathology. Avian Pathology. 2012; 41:3-9. [PubMed: 22845316]

Carrozza JH, Fredrickson TN, Prince RP, Luginbuhl RE. Role of desquamated epithelial cells in transmission of Marek's disease. Avian Diseases. 1973; 17:767-781. [PubMed: 4203252]

Chubb RC, Churchill AE. Precipitating antibodies associated with marek's disease. Veterinary Record. 1968; 83:4-7.

Cleveland RB, Cleveland WS, McRae JE, Terpenning I. STL: A seasonal-trend decomposition procedure based on loess. Journal of Official Statistics. 1990; 6:3-73.

Davison, F.; Nair, V., editors. Marek's Disease: An Evolving Problem. Elsevier Academic Press; 2004.

Davison F, Nair V. Use of Marek's disease vaccines: could they be driving the virus to increasing virulence? Expert Review of Vaccines. 2005; 4:77-88. [PubMed: 15757475]

Dudnikov, LA.; Witter, RL. A comparison of autologous and heterologous vaccination against Marek's disease. Proceedings of the 6th International Symposium on Mareks Disease, Current Progress on Marek's Disease Research; American Association of Avian Pathologists; Kennett Square, PA. 2001. p. 249-255.

Dunn JR, Gimeno IM. Current status of Marek's disease in the United States and worldwide based on a questionnaire survey. Avian Diseases. 2013; 57:483-490. [PubMed: 23901765]

Earn DJD, Rohani P, Bolker BM, Grenfell BT. A simple model for complex dynamical transitions in epidemics. Science. 2000; 287:667-670. [PubMed: 10650003]

Food Safety and Inspection Service. [Accessed: 2014-06-14] Post-mortem inspection. 2012. http:// www.fsis.usda.gov/wps/wcm/connect/6d982860-3c8d-4685-8068-6cffd00ae9ec/PHVtPost_Mortem_Inspection.pdf?MOD=AJPERES

Grunder AA, Gavora JS, Spencer JL, Turnbull JE. Prevention of Marek's disease using a filtered air positive pressure house. Poultry Science. 1975; 54:1189-1192.

Härdle, W. Applied Nonparametric Regression. Vol. 27. Cambridge Univ Press; 1990.

Havenstein GB, Ferket PR, Qureshi MA. Growth, livability, and feed conversion of 1957 versus 2001 broilers when fed representative 1957 and 2001 broiler diets. Poultry Science. 2003; 82:15001508.

Huang, JZ.; Stone, CJ. Nonlinear Estimation and Classification. Springer; 2003. Extended linear modeling with splines; p. 213-233.

Islam A, Walkden-Brown SW. Quantitative profiling of the shedding rate of the three Marek's disease virus (MDV) serotypes reveals that challenge with virulent MDV markedly increases shedding of vaccinal viruses. Journal of General Virology. 2007; 88:2121-2128. [PubMed: 17622613]

Jurajda V, Klimes B. Presence and survival of Marek's disease agent in dust. Avian Diseases. 1970; 14:188-190. [PubMed: 4985028]

King D, Page D, Schat KA, Calnek BW. Difference between influences of homologous and heterologous maternal antibodies on response to serotype-2 and serotype-3 Marek's disease vaccines. Avian Diseases. 1981; 25:74-81. [PubMed: 6268052]

Luginbuhl RE. The commercial production of specific-pathogen-free eggs and chickens: The evolution of an industry. Avian Diseases. 2000; 44:632-637. [PubMed: 11007011]

Meyer S, Steiner L. Daily livestock report. CME Group. 2011; 9:22.

Mooi FR, Van Loo IH, King AJ. Adaptation of Bordetella pertussis to vaccination: a cause for its reemergence? Emerging Infectious Diseases. 2001; 7:526. [PubMed: 11485646]

Morrow, C.; Fehler, F. Marek's disease: a worldwide problem. In: Davison, F.; Nair, V., editors. Marek's Disease: An Evolving Problem. Elsevier; 2004. p. 49-61. 
Nair V. Evolution of Marek's disease-a paradigm for incessant race between the pathogen and the host. The Veterinary Journal. 2005; 170:175-183. [PubMed: 16129338]

Okazaki W, Purchase HG, Burmester BR. Protection against Marek's disease by vaccination with a herpesvirus of turkeys. Avian Diseases. 1970; 14:413-429. [PubMed: 4913403]

Pitzer VE, Viboud C, Lopman BA, Patel MM, Parashar UD, Grenfell BT. Influence of birth rates and transmission rates on the global seasonality of rotavirus incidence. Journal of The Royal Society Interface. 2011:rsif20110062.

Pitzer VE, Viboud C, Simonsen L, Steiner C, Panozzo CA, Alonso WJ, Miller MA, Glass RI, Glasser JW, Parashar UD, et al. Demographic variability, vaccination, and the spatiotemporal dynamics of rotavirus epidemics. Science. 2009; 325:290-294. [PubMed: 19608910]

Powell PC, Lawn AM, Payne LN, Rennie M, Ross LJN. The effect of virus dose on the development of Marek's disease in two strains of chickens. Avian Pathology. 1980; 9:567-574. [PubMed: 18770298]

Purchase, HG. Clinical disease and its economic impact. In: Payne, LN., editor. Marek's Disease: Scientific Basis and Methods of Control. Boston: Martinus Nijhoff Publishing; 1985. p. 17-42.

Purchase HG, Okazaki W. Effect of vaccination with herpesvirus of turkeys (HVT) on horizontal spread of Marek's disease herpesvirus. Avian Diseases. 1971; 15:391-397. [PubMed: 4325826]

R Development Core Team. R: A Language and Environment for Statistical Computing. Vienna, Austria: R Foundation for Statistical Computing; 2012. ISBN 3-900051-07-0

Read, AF.; Mackinnon, MJ. Pathogen evolution in a vaccinated world. In: Stearns, SC.; Koella, JC., editors. Evolution in Health and Disease. Oxford University Press; 2008. p. 139-152.

Ricks CA, Avakian A, Bryan T, Gildersleeve R, Haddad E, Ilich R, King S, Murray L, Phelps P, Poston R, Witfill C, C W. In ovo vaccination technology. Advances in Veterinary Medicine. 1999; 41:495-515.

Rispens BH, van Vloten H, Mastenbroek N, Maas HJL, Schat KA. Control of Marek's disease in the Netherlands. I. Isolation of an avirulent Marek's disease virus (strain CVI 988) and its use in laboratory vaccination trials. Avian Diseases. 1972; 16:108-125. [PubMed: 4337307]

Rispens BH, van Vloten J, Maas HJ. Some virological and serological observations on Marek's disease: a preliminary report. British Veterinary Journal. 1969; 125:445-453. [PubMed: 4317990]

Rohani P, Earn DJD, Grenfell BT. Opposite patterns of synchrony in sympatric disease metapopulations. Science. 1999; 286:968-971. [PubMed: 10542154]

Sainsbury, D. Poultry health and management: chickens, turkeys, ducks, geese and quail. 4th edition. Oxford: Blackwell Science; 2000.

Sarma G, Greer W, Gildersleeve RP, Murray DL, Miles AM. Field safety and efficacy of in ovo administration of HVT + SB-1 bivalent Marek's disease vaccine in commercial broilers. Avian Diseases. 1995:211-217. [PubMed: 7677641]

Schat, KA.; Nair, V. Marek's disease. In: Saif, YM., editor. Diseases of Poultry. Twelfth Edition. Ames Iowa: Blackwell Publishing; 2008. p. 452-514.

Sharma, JM. Laboratory diagnosis. In: Payne, LN., editor. Marek's Disease: Scientific Basis and Methods of Control. Boston: Martinus Nijhoff Publishing; 1985. p. 151-175.

United States Department of Agriculture. USDA agricultural projections to 2020. Long-term Projections Report. 2011:81-89.

United States Department of Agriculture. Poultry Slaughter. 2014 May.:1-13. 2014.

Walkden-Brown SW, Islam A, Groves PJ, Rubite A, Sharpe SM, Burgess SK. Development, application, and results of routine monitoring of Marek's disease virus in broiler house dust using real-time quantitative PCR. Avian Diseases. 2013; 57:544-554. [PubMed: 23901774]

Welch TJ, Verner-Jeffreys DW, Dalsgaard I, Wiklund T, Evenhuis JP, Cabrera JAG, Hinshaw JM, Drennan JD, LaPatra SE. Independent emergence of Yersinia ruckeri biotype 2 in the United States and Europe. Applied and Environmental Microbiology. 2011; 77:3493-3499. [PubMed: 21441334]

Witter, RL. Principles of vaccination. In: Payne, LN., editor. Marek's Disease: Scientific Basis and Methods of Control. Boston: Martinus Nijhoff Publishing; 1985. p. 203-250. 
Witter, RL. Historic incidence of Marek's disease as revealed by condemnation statistics. In: Silva, RF.; Cheng, HH.; Coussens, PM.; Lee, LF.; Velicer, LF., editors. Current Research on Marek's disease. Pennsylvania: American Association of Avian Pathologists; 1996. p. 501-508.

Witter RL. Increased virulence of Marek's disease virus field isolates. Avian Diseases. 1997; 41:149_ 163. [PubMed: 9087332]

Witter RL, Sharma JM, Lee LF, Opitz HM, Henry CW. Field trials to test the efficacy of polyvalent Marek's disease vaccines in broilers. Avian Diseases. 1984; 28:44-60. [PubMed: 6326743] 


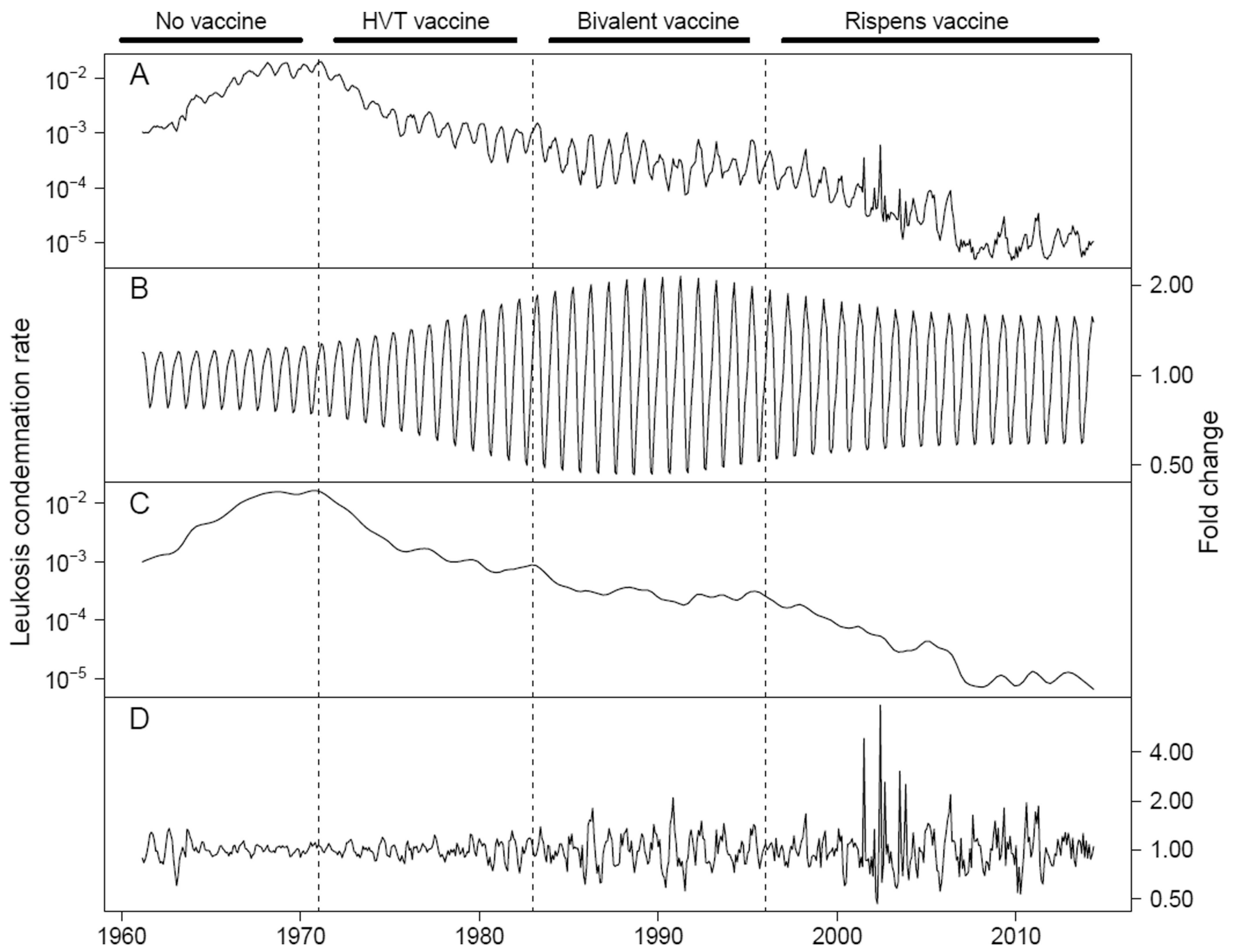

Figure 1.

STL analysis of the nationally aggregated leukosis condemnation rate in young birds over time. From top to bottom, (A) is the condemnation rate data, (B) is the fold change in leukosis condemnation that can be explained by the annual seasonal component, $(\mathrm{C})$ is the trend in the leukosis condemnation rate over time, and (D) is the remaining fold change difference between the data and the seasonal and trend components. The dotted lines indicate the approximate period at which new vaccines became available, and the bars at the top show the most recent vaccine available for use. We note that older generation vaccines (i.e. HVT, bivalent) continued to be used even after newer generation vaccines were introduced. In fact, the Rispens vaccine is only rarely used in broiler chickens even today. 


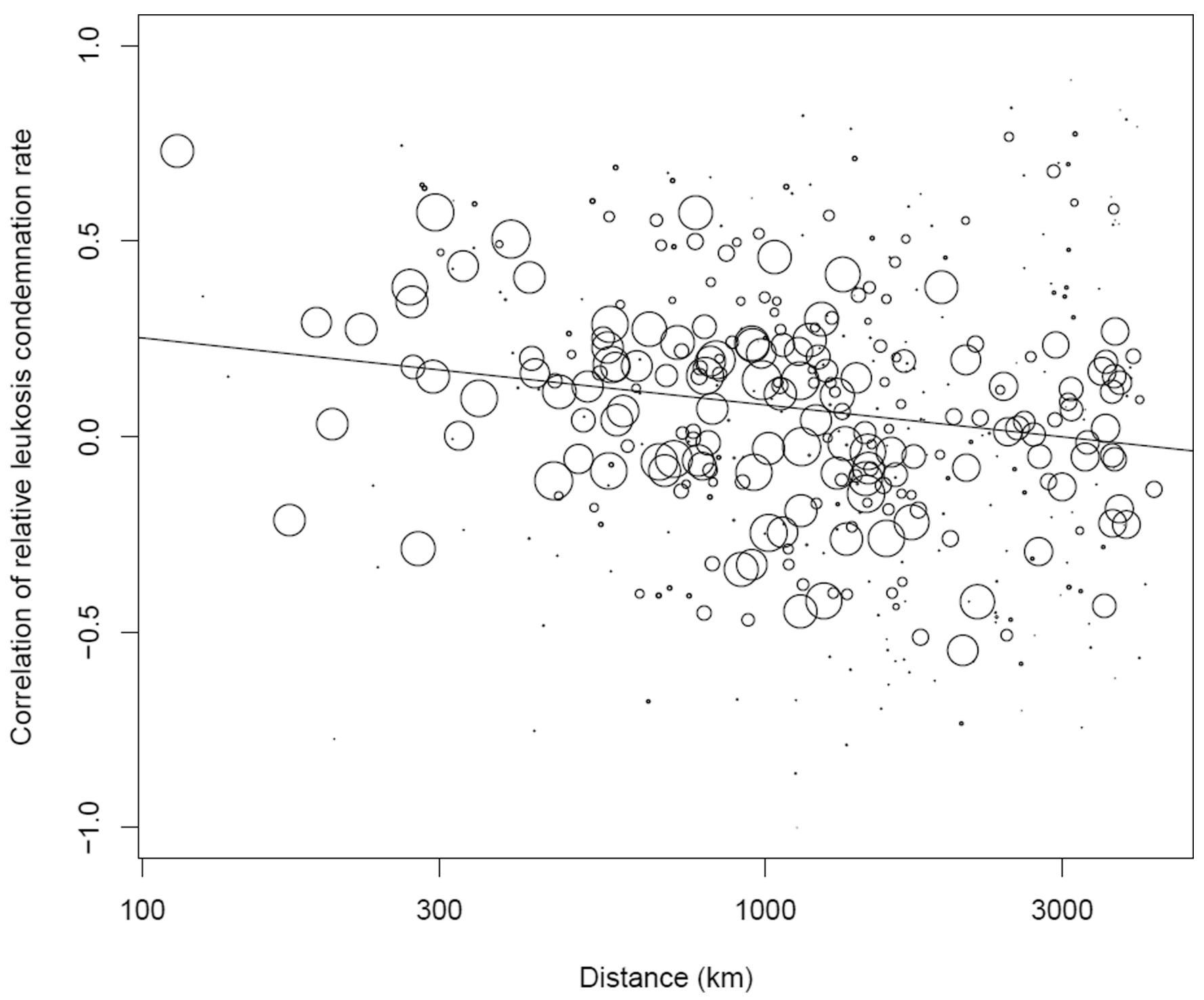

Figure 2.

Pairwise correlation coefficients in the relative leukosis condemnation rates by distance. Each circle shows the correlation between pairwise combinations of states. The size of the circle relates to the number of overlapping data points available with larger circles representing more overlapping data and thus better estimated correlation coefficients. As noted in the main text, a large number of overlapping data points is necessary to accurately estimate correlation coefficients and so only pairs of states with greater than 100 overlapping data points were used in our analysis. Nevertheless, all pairwise correlation coefficients are shown in this figure. The solid black line is the best-fit regression line. 


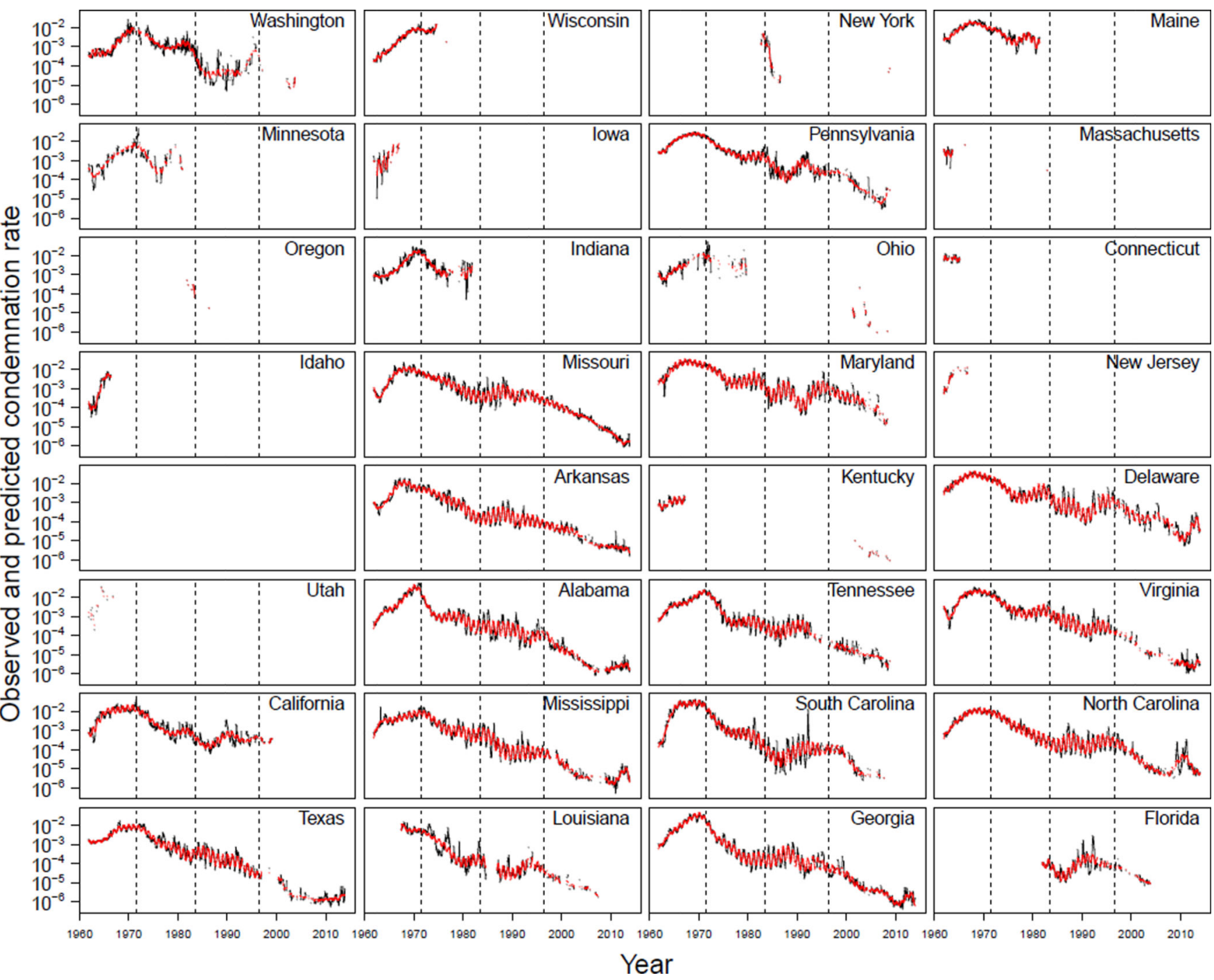

Figure 3.

The leukosis condemnation rate data (in black) and the fitted model values (in red). Each panel shows a different state. The dashed lines mark the approximate dates of vaccine introductions. 